\title{
Wie korrelieren BAKO 1-4- und H-LAD mit auditiven Verarbeitungsprozessen?
}

\author{
How do Results in BAKO 1-4 and H-LAD-test Correlate with Auditory \\ Processing?
}

Autoren

Institut
S. Brosch, R. Reiter, J. Imgrunt, N. Budde, T. Rinker

Sektion für Phoniatrie und Pädaudiologie der HNO Univ.-Klinik (Prof. Dr. G. Rettinger), Ulm

\section{Schlüsselwörter \\ - phonologische Bewusstheit \\ - auditive Verarbeitung \\ - BAKO 1-4 \\ O H-LAD \\ c FB-AVWS}

Korrespondenzadresse Prof. Dr. med. Sibylle Brosch Sektion für Phoniatrie und Pädaudiologie der HNO Univ.-Klinik Ulm Frauensteige 12 89075 Ulm

sibylle.brosch@uniklinik-ulm.de

\section{Zusammenfassung}

$\checkmark$

Hintergrund: Überprüft wird bei 75 Schulkindern der 1.-5. Klasse, welche Zusammenhänge zwischen basalen Fertigkeiten des LeseRechtschreiberwerbs, der Lautdifferenzierung und auditiven Funktionen bestehen.

Material und Methodik: Es werden periphere Hörtests und Hörprüfungen zur Erfassung auditiver Verarbeitungsprozesse, Tests zur Überprüfung der phonologischen Verarbeitung (BAKO 1-4; Basiskompetenzen für Lese- und Rechtschreibleistungen im 1.-4. Schuljahr; HLAD; Heidelberger Lautdifferenzierungstest) und der von den Eltern ausgefüllte Fragebogen (FBAVWS) ausgewertet.

Ergebnisse: BAKO 1-4 und H-LAD korrelieren erwartungsgemäß gut, was für eine reliable Testgüte spricht. Geschlechtsspezifische Unter-

\section{Einleitung \\ $\%$}

Die auditive Verarbeitungs- und Wahrnehmungsstörung (AVWS) wird in der ICD-10 unter F 20.20 kodiert. Eine normale kognitive Entwicklung (IQ mindestens 85) wird neben dem Ausschluss einer peripheren Hörstörung und einer Aufmerksamkeitsstörung vorausgesetzt [14-16]. Die American Speech Language Hearing Association (ASHA) definiert eine AVWS durch Defizite in der Tondifferenzierung, dem Richtungshören, der sequentiellen auditiven Analyse und dem Erkennen auditiver Signale unter Störschall $[25,26]$. Es wird aber auch die Meinung vertreten, dass die phonologische Bewusstheit, d.h. das Richten der Aufmerksamkeit auf formale Aspekte der Sprache (Reimen, Lautunterscheidung, Phonemerkennung usw.), ebenfalls einen bedeutsamen auditiven Faktor bei Kindern im Spracherwerb darstellt $[4,16]$. Gesicherte Angaben zur Prävalenz der AVWS existieren nicht. Bamiou et al. gehen schiede bestehen nicht. Außerdem zeigt diese Untersuchung, dass der FB-AVWS zwar die symptomorientierte Anamnese bei Kindern mit V.a. AVWS erweitern kann, als diagnostisches Instrument jedoch nicht einsetzbar ist. Die Hörtests erlauben ebenso wenig wie der BAKO 1-4 und HLAD als alleiniges Testinventar eine Unterscheidung zwischen den vier Diagnosen „AVWS“, „LRS“, „Sprachentwicklungsstörung“ und „Störung der phonologischen Bewusstheit“".

Schlussfolgerungen: Die Lautdifferenzierungsfähigkeit kann nicht unmittelbar auditiven Funktionen zugeordnet werden. Eine individuelle, therapiesteuernde Diagnostik muss für jedes Kind mit auffälliger Laut- und/oder Schriftsprachentwicklung gefordert werden, die dem komplexen Ursachengefüge gerecht wird und letztendlich nur interdisziplinär geleistet werden kann.

von einer Prävalenz von 7\% aus [2]. Übereinstimmung herrscht dahingehend, dass das männliche Geschlecht etwa doppelt so häufig betroffen ist [16]. Die unterschiedlichen Häufigkeitsangaben rühren daher, dass es noch immer große Unsicherheiten bei den Diagnosekriterien einer AVWS und zudem Überlappungen mit anderen Störungsbildern wie der Lese-Rechtschreibstörung (LRS) gibt. Klicpera et al. postulieren, dass ein deutlicher Zusammenhang zwischen der Fähigkeit der phonologischen Rekodierung im Arbeitsgedächtnis und Lese-Rechtschreibschwierigkeiten [12] besteht. Nickisch et al. [17] fanden in ihrer Multizenterstudie eine schwache, signifikante Korrelation zwischen der Skala „auditive Diskrimination" des FB-AVWS und den H-LAD Subtests „Kinästhetik/Lautidentifikation“ sowie „Lautanalyse“. Rosenauer et al. [21] haben 282 Kinder mit einem Durchschnittsalter von 8;6 Jahren untersucht und in der Gesamtstichprobe ebenfalls nur schwache Korrelationen zwischen 
der Skala „auditive Diskrimination“ sowie „auditives Gedächtnis“ des Fragebogens und dem Gesamtergebnis des H-LAD sowie seiner Untertests 1B „Differenzierung von Konsonanten“ und „Analyse und Differenzierung von Konsonantenhäufung im Anlaut" gefunden. Ptok \& Buller [19] haben mit einer Faktorenanalyse bei 62 Kindern herausgestellt, dass der BAKO 1-4 lediglich auf zwei Faktoren lädt. Mehrere Studien haben gezeigt, dass die Sprachwahrnehmung und -verarbeitung eine zentrale Rolle für den erfolgreichen Schriftspracherwerb hat [22-25], weshalb Kinder mit Störungen beim Einstieg in den Lese- oder Schriftsprachlernprozess nicht nur hinsichtlich ihrer sprachlichen, sondern auch ihrer auditiven Funktionen getestet werden. Aus diesem Zusammenhang und den in der wissenschaftlichen Literatur noch immer sehr widersprüchlichen Befunden leiten sich die Ziele dieser Studie ab:

- Zusammenhänge zwischen dem BAKO 1-4/H-LAD und sprachfreien sowie sprachgebundenen auditiven Testverfahren (Richtungsgehör, dichotischer Diskriminationstest, Hören im Störschall usw.)

- Zusammenhang mit einem Elternfragebogen zur AVWS

- Geschlechtsspezifische Unterschiede beim BAKO 1-4 und H-LAD

- Konsequenzen für die Praxis.

\section{Material und Methoden \\ $\checkmark$}

Stichprobenbeschreibung

In diese retrospektive Studie wurden 75 Schulkinder mit Verdacht auf bzw. gesicherte LRS und/oder AVWS eingeschlossen. Sie haben sich zwischen 2005 und 2007 an der HNO-Universitätsklinik Ulm, Sektion Phoniatrie und Pädaudiologie, vorgestellt. Die meisten Kinder wiesen schulische Leistungsschwierigkeiten und Auffälligkeiten im Spracherwerb auf. Innerhalb dieses Patientenkollektives waren $69,3 \%(n=52)$ männlich und $30,7 \%(n=23)$ weiblich Die Altersspanne lag zwischen $6 ; 0$ und 11;0 Jahren (1.-5. Klasse). Das Durchschnittsalter betrug 8;7 Jahre $(\mathrm{SD} \pm 1 ; 1)$.

\section{Ein- und Ausschlusskriterien}

Einschlusskriterien waren, dass die Kinder eingeschult, nicht jünger als 6 und nicht älter als 11 Jahre alt waren und keine allgemeinen Entwicklungsverzögerungen aufwiesen. Der IQ-Wert lag dabei im Normbereich $(I Q \geq 85)$. Eine LRS wurde dann als gesichert angenommen, wenn die Lese- und Rechtschreibleistungen in standarisierten Testverfahren deutlich unter dem Intelligenzniveau lagen. Als weiteres Kriterium musste die pancochleäre Hörschwelle im Tonaudiogramm unter $25 \mathrm{~dB} \mathrm{HL}$ liegen.

\section{Testverfahren und -auswahl}

Die Diagnose „AVWS“ stützte sich auf mindestens 2 auffällige audiometrische Testparameter (siehe auch Studie von Wohlleben et al., 2007), Einschränkungen in der auditiven Merkfähigkeit (gemessen anhand nachgesprochener Sätzen aus dem Heidelberger Sprachentwicklungstest [8] und Defizite in der phonologischen Verarbeitung im Sinne der Lautanalyse und -differenzierung (gemessen anhand des H-LAD und BAKO 1-4). Die Hördiagnostik bestand aus einer Hörschwellendiagnostik und objektiven Testverfahren (Tympanometrie, Stapediusreflexmessung, Ableitung der Distorsionsprodukte otoakustischer Emissionen). Zur hörverarbeitenden Diagnostik wurden sprachfreie und sprachgebundene Hörtests herangezogen (dichotischer Diskriminationstest nach Uttenweiler bzw. Feldmann, Prüfung des Richtungshörens, Bestimmung der Ordnungsschwelle und Sprachaudiometrie $(65 \mathrm{~dB})$ im Störschall $(60 \mathrm{~dB}))$.

\section{FB-AVWS}

Der „Anamnese- und Beurteilungsbogen zur Erfassung von Auditiven Verarbeitungsstörungen" (FB-AVWS) wurde von der Deutschen Gesellschaft für Phoniatrie und Pädaudiologie (DGPP) entwickelt [1]. Er umfasst folgende sieben Subklassen: Allgemeine Fragen (AF), Auditive Diskrimination (DI), Richtungsgehör (RI), Selektionsfähigkeit/Hören im Störschall (SE), Auditives Gedächtnis (GD), und Geräuschüberempfindlichkeit (GÜ). Die Fragen werden nach der vierstufigen Likert-Skala eingeschätzt [Bewertung von 0 (keine Probleme) bis 3 (sehr viele Probleme)]. Außerdem wurde die Spalte „weiß nicht“ eingefügt [9]. Der Fragebogen wurde von den Eltern ausgefüllt (Fragebogen im Anhang).

\section{BAKO 1-4}

Der Test der Basiskompetenzen für Lese-Rechtschreibleistungen in den ersten 4 Grundschuljahren (BAKO 1-4) untersucht die Fähigkeit, die Lautstruktur der gesprochenen Sprache zu analysieren und mit den Lauten der Sprache umgehen zu können. Der Test besteht aus 74 Items in sieben verschienen Subtests: Pseudowortsegmentierung (PWS), Vokalersetzung (VE), Restwortbestimmung (RWB), Phonemvertauschung (PV), Lautkategorisierung (LK), Vokallängenbestimmung (VLB) und Wortumkehr (WU).

\section{H-LAD}

Der Heidelberger Lautdifferenzierungstest (H-LAD) dient der Uberprüfung der Lautanalyse und dem Erfassen einer Differenzierungsschwäche bei Kindern. Es existieren Normwerte von der ersten bis zur vierten Klasse. Er besteht aus den Subtests: Auditive Phonemdifferenzierung und „Kinästhetik/ Lautidentifikation bzw. „Analyse und Differenzierung von Konsonantenanhäufung im Anlaut“.

Ein positives Votum der Ethikkommission der Universität Ulm für die Durchführung dieser Untersuchung liegt vor. Alle Eltern der Studienteilnehmer wurden schriftlich über das Studienprojekt informiert.

\section{Ergebnisse}

$\nabla$

Das Geschlechterverhältnis entspricht in etwa einer Relation von 2:1. In o Tab. 1 sind die nominalen und prozentualen Verteilungen der angewandten Testverfahren dargestellt. BAKO 1-4 und H-LAD wurde bei allen 75 Studienteilnehmern durchgeführt, der FB-AVWS wurde von 41 Eltern ausgefüllt.

$71 \%$ der Studienkinder $(n=53)$ hatten Auffälligkeiten in der phonologischen Verarbeitung (diagnostiziert anhand des H-LAD und BAKO 1-4). Am zweithäufigsten waren eine Sprachentwicklungsstörung (diagnostiziert anhand des phoniatrischen Be-

\begin{tabular}{|c|c|c|c|}
\hline & ВАKO & H-LAD & FB-AVWS \\
\hline männlich & 52 (69\%) & $52(69 \%)$ & $31(41,4 \%)$ \\
\hline weiblich & $23(31 \%)$ & $23(31 \%)$ & $10(13,3 \%)$ \\
\hline Gesamt & $75(100 \%)$ & $75(100 \%)$ & $41 / 75(54,7 \%)$ \\
\hline
\end{tabular}




\begin{tabular}{|c|c|c|c|c|c|c|c|c|}
\hline \multirow[t]{3}{*}{ H-LAD } & \multicolumn{8}{|c|}{ Bako 1-4 } \\
\hline & PWS & VE & RWB & PV & LK & VLB & wu & GESAMT \\
\hline & T-W & T-W & T-W & T-W & T-W & T-W & T-W & $T-W$ \\
\hline Subtest 1 A T -W & n.s. & ++ & ++ & ++ & n.s. & n.s. & n.s. & + \\
\hline Subtest 1 B T-W & ++ & ++ & ++ & n.s. & + & n.s. & + & ++ \\
\hline Subtest $2 \mathrm{~T}-\mathrm{W}$ & ++ & ++ & ++ & ++ & ++ & n.s. & + & ++ \\
\hline Gesamt T-W & ++ & ++ & ++ & ++ & ++ & n.s. & + & ++ \\
\hline \multicolumn{9}{|c|}{$\begin{array}{l}\text { n. s. nicht signifikant }(p>0,05) \\
+ \text { signifikant }(p<0,05) \\
++ \text { hochsignifikant }(p<0,01)\end{array}$} \\
\hline
\end{tabular}

\begin{tabular}{|c|c|c|c|c|}
\hline \multirow[t]{2}{*}{ Geschlecht } & \multirow[t]{2}{*}{$n$} & \multicolumn{3}{|c|}{ T-Wert Bako 1-4 } \\
\hline & & Mittelwert & Min & $\operatorname{Max}$ \\
\hline männlich & 52 & 41,5 & 21,0 & 67,0 \\
\hline weiblich & 23 & 43,4 & 28,0 & 58,0 \\
\hline \multirow[t]{2}{*}{ Geschlecht } & $n$ & \multicolumn{3}{|c|}{ T-Wert H-LAD } \\
\hline & & Mittelwert & Min & Max \\
\hline männlich & 52 & 45,3 & 27,0 & 65,0 \\
\hline weiblich & 23 & 44,1 & 23,0 & 60,0 \\
\hline
\end{tabular}

fundes; $n=41 ; 55 \%$ ) bzw. LRS (diagnostiziert anhand standardisierter Lese-Rechtschreibetests; $(n=30 ; 40 \%$ ). Auf einem ähnlich hohen Niveau lag die Diagnose AVWS (diagnostiziert anhand auditiver Teste; $\mathbf{n}=27 ; 36 \%$ ).

Zunächst wurde eine Korrelation nach Pearson zum Vergleich der T-Werte zwischen H-LAD und BAKO 1-4 durchgeführt. Die Ergebnisse wiesen eine statistisch signifikante Korrelation $(p<0,0001 ; r=0,490)$ zwischen den beiden Testen auf. Um Aussagen über die Beziehung zwischen den einzelnen Leistungen, die mit H-LAD und BAKO 1-4 untersucht werden, zu machen, wurden die einzelnen Untertests von BAKO 1-4 und H-LAD korreliert. Die Zusammenhänge zwischen den T-Werten sind in Tab. 2 dargestellt.

Somit ergaben sich eine ganze Anzahl signifikanter statistischer Korrelationen zwischen einzelnen Untertests von BAKO 1-4 und H-LAD. Lediglich bei der Vokallängenbestimmung war keine Korrelation vorhanden. Die Ergebnisse sowohl im BAKO 1-4 als im H-LAD zeigten keine signifikanten geschlechtsspezifischen Unterschiede (c Tab. 3).

Wie o Tab. 3 zeigt, liegt der Mittelwert beim BAKO 1-4 bei den Jungen mit 41,5 $(\mathrm{SD} \pm 9,4)$ etwas niedriger als bei den Mädchen $(43,4 ; S D \pm 7,9)$. Die Extremwerte sind vergleichbar. Im Gegensatz zum BAKO 1-4, lag der Mittelwert des T-Wertes beim $\mathrm{H}$ LAD bei den Jungen mit 45,3 ( $S D \pm 9,0)$ etwas höher als bei den Mädchen (44,1; SD $\pm 8,7)$. Auch die Extremwerte lagen bei den Mädchen etwas niedriger ( $\square$ Tab. 4).

Daraufhin wurde überprüft, inwieweit Zusammenhänge zwischen einer LRS und auditiven Funktionen bestanden. Dabei zeichnete sich lediglich bei der Verdachtsdiagnose LRS eine schwache, positive signifikante Korrelation zur Höhe der Ordnungsschwelle ab. Alle übrigen Hörergebnisse korrelierten nicht signifikant.

Im Weiteren wurden die Korrelationen zwischen der Diagnose AVWS und dem anamnestischen Fragebogen (FB-AVWS) errechnet. Im Einzelnen korrelierten die Fragen des FB-AVWS DI 4 (Kategorie „auditive Diskrimination“; Frage 4) und SE 5 (Kategorie
Tab. 5 Korrelationen nach Pearson zwischen der AVWS-Diagnose und dem FB-AWWS.

\begin{tabular}{llll} 
FB-AVWS & $\mathbf{n}$ & Korrelationskoeffizient & Signifikanz \\
DI 4 & 40 & 0,331 & 0,037 \\
SE 5 & 40 & 0,331 & 0,037 \\
GD 1 & 40 & 0,443 & 0,004 \\
GD 2 & 40 & 0,475 & 0,002 \\
\hline
\end{tabular}

Erläuterung zu den Abkürzungen: Dl: Auditive Diskrimination;

SE: Selektionsfähigkeit/Hören im Störschall; GD: Auditives Gedächtnis

„Selektionsfähigkeit/Hören im Störschall“; Frage 5), sowie die Fragen zum auditiven Gedächtnis GD1 (Kategorie „auditives Gedächtnis“; Frage 1) und GD 2 (Kategorie „auditives Gedächtnis“; Frage 2) signifikant mit der Diagnose AVWS (॰ Tab. 5).

Bei Kindern mit einer eingeschränkten auditiven Merkfähigkeit, einer auditiven Filterschwäche und V.a. AVWS konnte keine statistische Berechnung durchgeführt werden, da die Fallzahlen zum Errechnen des p-Wertes zu gering waren.

Bei der Korrelation zwischen den beiden H-LAD Untertesten und den auditiven Hörtests findet sich nur eine Korrelation zwischen der Ordnungsschwelle und dem Subtest 2 (Lautanalyse) $(\mathrm{r}=-0,270 ; \mathrm{p}=0,041)$. Darüber hinaus wurde festgestellt, dass die Ergebnisse des dichotischen Hörtests signifikant mit den Ergebnissen des Richtungshörens korrelierten ( $r=0,326$; $\mathrm{p}=0,006$ ).

Die Korrelationen zwischen den Untertesten im BAKO 1-4 und den Hörtests sind in 0 Tab. 6 dargestellt:

Dabei korrelierten die Ergebnisse des dichotischen Hörtests sowohl mit dem Untertest im BAKO 1-4 „Vokalersetzung“ signifikant $(r=0,285 ; p=0,016)$, als auch mit dem BAKO-Untertest "Lautkategorisierung" $(r=0,251 ; p=0,035)$. Beim Richtungshören ergab sich eine signifikante Korrelation mit dem BAKOUntertest „Wortumkehr“ ( $r=0,281 ; p=0,018)$. Das Testverfahren zum Hören im Störschall korrelierte ebenfalls signifikant mit dem BAKO-Untertest „Wortumkehr“ ( $r=0,247 ; p=0,043)$. Die Korrelationskoeffizienten sind allerdings relativ gering. 


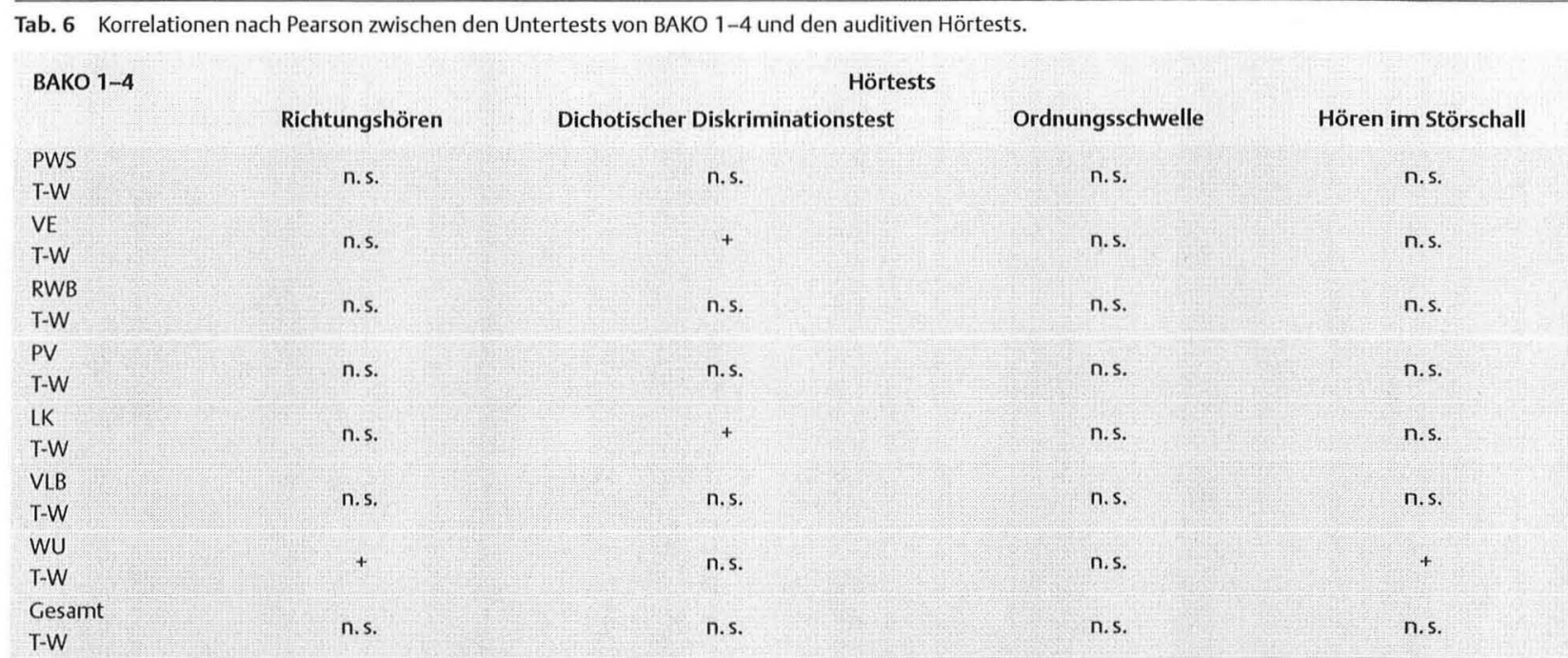

Erläuterungen zu den Abkürzungen: PWS: Pseudowortsegmentierung; VE: Vokalersetzung: RWB: Restwortbestimmung; PV: Phonemvertauschung: LK: Lautkategorisierung: VLB: Vokallängenbestimmung; WU: Wortumkehr

Tab. 7 Korrelation nach Pearson zwischen dem Gesamt-T-Wert von H-LAD und dem FB-AVWS.

$\begin{array}{llll}\text { FB-AVWS } & \mathbf{n} & \text { Korrelationskoeffizient } & \text { Signifikanz } \\ \text { AF 5 } & 38 & -0,333 & 0,041 \\ \text { GD 6 } & 39 & -0,368 & 0,021 \\ \text { GD } 8 & 38 & -0,387 & 0,017\end{array}$

Erläuterung zu den Abkürzungen: AF: Allgemeine Fragen; CD: Auditives Cedächtnis

Tab. 8 Korrelation nach Pearson zwischen dem Gesamt-T-Wert von BAKO 1-4 und dem FB-AVWS.

$\begin{array}{llll}\text { FB-AVWS } & \mathbf{n} & \text { Korrelationskoeffizient } & \text { Signifikanz } \\ \text { AF 5 } & 38 & -0,376 & 0,020 \\ \text { DI 1 } & 40 & -0,312 & 0,050 \\ \text { GD 1 } & 40 & -0,342 & 0,031 \\ \text { GD 2 } & 40 & -0,386 & 0,014 \\ \text { GD 3 } & 40 & -0,426 & 0,006 \\ \text { GD 6 } & 39 & -0,416 & 0,008 \\ \text { GD 8 } & 38 & -0,457 & 0,004 \\ \text { GD 9 } & 40 & -0,469 & 0,002\end{array}$

Erläuterung der Abkürzungen in der Tabelle: AF: Allgemeine Fragen; DI: Auditive Diskrimination; GD: Auditives Gedächtnis

Zwischen dem dichotischen Diskriminationstest und den Elternangaben aus dem FB-AVWS zeigten sich statistisch signifikante Zusammenhänge zu den Kategorien „Richtungsgehör“, „auditives Gedächtnis“" sowie "Geräuschüberempfindlichkeit" des Fragebogens. Die übrigen Hörtests korrelierten nicht mit dem FB-AVWS.

- Tab. 7 stellt die statistischen Zusammenhänge der Gesamt TWerte im H-LAD bzw. FB-AVWS dar.

Dabei ergaben sich zwischen dem H-LAD und den sieben Kategorien des FB-AVWS statistisch signifikante Korrelationen bei den zwei Kategorien „allgemeine Fragen“ und „auditives Gedächtnis“.

Aus 0 Tab. 8 gehen die statistischen Zusammenhänge der Gesamt T-Werte im BAKO 1-4 bzw. FB-AVWS hervor:
Der BAKO 1-4 korrelierte insgesamt hoch mit der Kategorie „auditives Gedächtnis des FB-AVWS. Als Einzelergebnisse sind hierbei folgende hervorzuheben: Signifikante, schwache Korrelationen fanden sich zwischen der Frage AF 5 (Kategorie „allgemeine Fragen“") und der Frage DI 5 (Kategorie „auditive Diskrimination“) sowie dem Gesamt-T-Wert im BAKO 1-4. Die überwiegende Anzahl der Fragen der Kategorie „auditives Gedächtnis“ des FB-AVWS korrelierte ebenfalls signifikant mit dem GesamtT-Wert des BAKO 1-4. Die Fragen des FB-AVWS GD 1-2 (Kategorie „auditives Gedächtnis“, korrelierten signifikant mit dem Gesamt-T-Wert von BAKO 1-4.

\section{Diskussion}

In dieser Studie wurde der Zusammenhang zwischen dem BAKO 1-4, H-LAD und auditiven Leistungen untersucht. Dabei wurden sprachfreie und sprachgebundene Hörtests eingesetzt. Die wesentlichen Ergebnisse waren, dass sich der FB-AVWS nicht für ein alleiniges AVWS Screening eignet. Der BAKO-1-4 überprüft die phonologische Verarbeitung im Sinne der Lautanalyse und -differenzierung deutlich differenzierter als der HLAD. Die auditive Diagnostik ergab keine diagnosespezifischen Unterschiede zwischen einer AVWS, einer LRS, einer Sprachentwicklungsstörung oder Störung der phonologischen Verarbeitung. Dasselbe gilt für den BAKO 1-4 und H-LAD,

Kummer et al. [13] haben 84 Vorschulkinder mittels FB-AVWS untersucht und mit Untertests des Heidelberger-Vorschulscreenings (HVS) sowie zwei Untertests des Heidelberger Sprachentwicklungstests (HSET) verglichen. Statistisch schwache, signifikante Korrelationen ergaben sich zwischen der FB-Skala „auditive Diskrimination“ und den HVS-Untertests „expressive Lautanalyse“, „Artikulomotorik“ sowie „auditives Gedächtnis“. Insgesamt bestätigen diese Ergebnisse auch die Einschätzung von Prelic et al. [18], die konstatiert haben, dass der FB-AVWS die Palette diagnostischer Maßnahmen sicherlich ergänzt, sich aber nicht für ein alleiniges Screening eignet. Ähnliche Resultate ergab die vorliegende Untersuchung auch beim Vergleich zwischen dem H-LAD und dem FB-AVWS: Hier zeigten sich statistisch signifikante Korrelationen lediglich bei den zwei Katego- 
rien „allgemeine Fragen“ und „auditives Gedächtnis“. Nickisch et al. [17] fanden in ihrer Multizenterstudie bei 113 Schülern der 2. und 4. Klassenstufen ebenfalls lediglich schwache, signifikante Korrelationen zwischen der Skala „auditive Diskrimination“ des FB-AVWS und den H-LAD Subtests „Kinästhetik/ Lautidentifikation" sowie „Lautanalyse“. Rosenauer et al. [21] haben 120 Mädchen und 162 Jungen mit einem Durchschnittsalter von 8;6 Jahren untersucht und in der Gesamtstichprobe nur schwache Korrelationen zwischen der Skala „auditive Diskrimination“ sowie „auditives Gedächtnis“ des Fragebogens und dem Gesamtergebnis des H-LAD sowie seiner Untertests 1B „Differenzierung von Konsonanten" und „Analyse und Differenzierung von Konsonantenhäufung im Anlaut" gefunden. Mit dem Prozentrang der Rechtschreibetests korrelierte in der $\mathrm{Ge}$ samtstichprobe die überwiegende Anzahl der Items aus der Skala „auditives Gedächtnis“ schwach bis mäßig.

Der FB-AVWS zeigte einige signifikante statistische Zusammenhänge zum dichotischen Diskriminationstest und den Kategorien „Richtungsgehör“, „auditives Gedächtnis“ sowie „Geräuschüberempfindlichkeit“. Die übrigen auditiven Hörtests korrelieren nicht mit dem FB-AVWS. Dies ist plausibel erklärbar, da der dichotische Diskriminationstest $u$. a. den auditiven Merkspeicher überprüft und somit statistische Zusammenhänge zu anamnestischen Angaben, das auditive Gedächtnis betreffend, nicht unerwartet sind. Das Richtungsgehör gelingt nur unter der Voraussetzung beidohrigen Hörens, welches auch eine Voraussetzung für den dichotischen Diskriminationstest ist. Geräuschüberempfindlichkeit entsteht $u$. a. bei einem insuffizienten efferenten Hörsystem mit verminderter lateraler Hemmung [5-7] und dadurch bedingter reduzierter Störschallunterdrückung. Zusammenhänge zu Auffälligkeiten im dichotischen Diskriminationstest sind bisher nicht berichtet worden und bedürfen einer weiteren Überprüfung an einem deutlich größeren Kollektiv, zumal diese Kategorie nicht mit der Sprachaudiometrie im Störschall korrelierte, was im Falle einer reduzierten Störschallunterdrückung zu erwarten gewesen ware.

BAKO 1-4 und H-LAD überprüfen Aspekte der phonologischen Verarbeitung, so verwundert es nicht, dass die Korrelationsanalyse beim Vergleich der T-Werte eine signifikante Korrelation ergab. Geschlechtsspezifische Unterschiede zeigten sich nicht. Der BAKO 1-4 korrelierte unterschiedlich stark mit Fragen zum auditiven Gedächtnis des FB-AVWS. Dieses Ergebnis ist kongruent mit Angaben aus der Literatur, die belegen, dass das auditive Arbeitsgedächtnis eine wesentliche Voraussetzung für das schnelle Rekodieren aus dem phonologischen Arbeitsspeicher darstellt $[3,11,20]$. Es errechneten sich keine korrelativen Zusammenhänge zwischen den einzelnen Diagnosetypen. Lediglich die Höhe der Ordnungsschwelle war schwach positiv signifikant mit der Verdachtsdiagnose einer LRS korreliert. Zu diesen Ergebnissen liegen aus der Literatur keine Vergleichsdaten vor. Bei der Korrelation zwischen den beiden H-LAD Untertesten und den auditiven Hörtests fand sich nur eine signifikante Korrelation zwischen der (sprachfreien) Ordnungsschwelle und dem Subtest zur Lautanalyse, in dem das Kind aufgefordert wird, die ersten beiden Buchstaben eines Wortes zu benennen. Dieses Ergebnis bestätigt die empirische Erfahrung, dass sich auch die Fähigkeit zur Lautdifferenzierung nicht unmittelbar auditiven Funktionen zuordnen lässt. Beim dichotischen Diskriminationstest stellte sich eine signifikante Korrelation mit dem H-LAD-Untertest „Vokalersetzung“ sowie der „Lautkategorisierung“ heraus, das Richtungshören und die Sprachdiskrimination im Störschall waren signifikant mit dem Untertest „Wortumkehr" korreliert. Die gefundenen eher geringen statistischen $\mathrm{Zu}$ sammenhänge beider Testinventare mögen mit eine Erklärung dafür sein, dass ein reines Training auditiver Funktionen in der VergangenheitkeineVerbesserungderLese-undRechtschreibleistungen nachweisen konnte $[10,26]$.

\section{Abstract}

\section{How do Results in BAKO 1-4 and H-LAD-test Correlate with Auditory Processing?}

\%

Objective: An increasing number of children seem to have a deficiency in Central Processing as a reason for reading and writing difficulties (RWD) and attention deficit disorder (ADD). There is often also a delay in phonological consciousness. A test would therefore be useful to distinguish children with RWD and ADD.

Material and Methods: This study involved 75 consecutive schoolchildren from the $1^{\text {st }}$ to $5^{\text {th }}$ classes (ages 6-11), and was carried out at the Department of Phoniatric-Paediatric Audiology at the University ENT Clinic Ulm. Various tests were undertaken: hearing tests for peripheral hearing, central auditory tests, tests of phonological competence and a questionnaire completed with the parents. Statistical calculations were used to verify correlations with clinical findings and the questionnaire.

Results: BAKO 1-4 and H-LAD test phonological awareness and the T-values showed a good correlation, as expected. These are therefore reliable tests. No gender specific differences could be found. The BAKO 1-4 test was better statistically and this confirms other groups' findings. The questionnaire is not ready to be used for diagnosis. It is not possible to distinguish different diagnoses (central auditory processing disorder, writing difficulties, delayed speech acquisition and delay in phonological consciousness) by means of auditory tests.

Conclusions: This means that the capacity to distinguish phonemes is not directly correlated to central auditory functions. Equally, one can also say that not every child with such problems has deficits in central auditory processing which require therapy. They all require an interdisciplinary management tailored to their own needs and underlying causes.

\section{Literatur}

1 AG AVWS der Deutschen Gesellschaft für Phoniatrie und Pädaudiologie (DGPP). Anamnesebogen zur Erfassung Auditiver Verarbeitungsund Wahrnehmungsstörungen (FB-AVWS)http://www.dgpp.de/Profi/ Sources/FragAVWS.pdf [accessed 18-1-2009]

2 Bamiou DE, Musiek FE, Luxon LM. Aetiology and clinical presentations of auditory processing disorders - a review. Arch Dis Child 2001; 85 361-365

3 Berninger VW, Raskind W, Richards T, Abbott $R$, Stock P. A multidisciplinary approach to understanding developmental dyslexia within working-memory architecture: genotypes, phenotypes, brain, and instruction. Dev Neuropsychol 2008; 33: 707-744

4 Bus AG, Van lizendoorn MH. Phonological awareness and early reading: A meta-analysis of experimental training studies. J educ psychol 1999; 91: $403-414$

5 Esser G. Differenzierung von Schallempfindungsstörungen durch vergleichende Stapedius-Reflexaudiometrie. Med Habilitationsschrift, Universität Düsseldorf; 1976

6 Esser G, Anderski C, Birken A, Breuer E, Cramer B, Eisermann E, Kulenkampff $H$, Schröer M, Schunicht $R$, Toro La Roche M. Auditive Wahrnehmungsstörungen und Fehlhörigkeit bei Kindern im Schulalter. Sprache Stimme Gehör 1987; 11: 10-16 
7 Esser G, Schunicht R, Hockauf H. Diagnostik der Fehlhörigkeit bei Kindern. In: Mortier W, Hrsg. Moderne Diagnostik und Therapie bei Kindern. 1. Auflage, Grosse, Berlin; 1984; 94-98

8 Grimm H, Schöler H. Der Heidelberger Sprachentwicklungstest H-S-ET. Hogrefe, Göttingen; 1978

9 Heuckmann C, Nickisch A. Normierung des anamnesebogens zur Erfassung Auditiver Verarbeitungs- und Wahrnehmungsstörungen (AVWS). In: Kruse E, Gross M, Hrsg. Aktuelle phoniatrisch - pädaudiologische Aspekte 2003/2004. 1. Auflage, Median, Heidelberg; 2003; 296-299

10 Keilmann A, Wintermeyer M. Is a Specialised Training of Phonological Awareness Indicated in Every Preschool Child? Folia Phoniatr Logop 2008; 60: 73-79

11 Kibby MY. There are Multiple Contributors to the Verbal Short-Term Memory Deficit in Children with Developmental Reading Disabilities. Child Neuropsychol 2009; 15: 1-22

12 Klicpera C, Schabmann A, Gasteiger-Klicpera B. Ursachen der LeseRechtschreibschwierigkeiten. In: Klicpera C, Schabmann A, GasteigerKlicpera B Hrsg. Legasthenie, 2. Auflage, Reinhardt, München; 2007 160-191

13 Kummer P, Preclik M, Döllinger M, Rosanowski F, Eysholdt U. Zusammenhang zwischen den Angaben im AVWS-Anamnesebogen der DGPP und den Ergebnissen des Heidelberger Vorschulscreeningshttp://www.egms.de/en/meetings/dgpp2006/06dgpp07.shtml [accessed 25-3-2009]

14 McFarland DJ, Cacace AT. Modality Specificity as a Criterion for Diagnosing Central Auditory Processing Disorders. Am J Audiol 1995; 4 36-48

15 McFarland DJ, Cacace AT. Modality specificity of auditory and visual pattern recognition: implications for the assessment of central auditory processing disorders. Audiology 1997; 36: 249-260

16 Nickisch A, Gross $M$, Schonweiler R, Uttenweiler V, am Zehnhoff-Dinnesen A, Berger R, Radu HJ, Ptok M. Auditive-Verarbeitungs- und Wahrnehmungsstörungen: Konsensus- Statement der Deutschen Geselschaft für Phoniatrie und Pädaudiologie. HNO 2007; 55 : 61-72

17 Nickisch A, Kiese-Himmel C, Schonweiler R, Gross M, Radu HJ. Zusammenhänge zwischen dem „Anamnestischen Erhebungs- und Beobachtungsbogen für auditive Verarbeitungs- und Wahrnehmungsstörungen" (AVWS) und dem „Heidelberger Lautdifferenzierungstest“ (H-LAD). Laryngo Rhino Otol 2005; 84: 487-492
18 Preclik $M$, Rosanowski F, Döllinger $M$, Eysholdt $U$, Kummer P. Anamnesebogen für auditive Verarbeitungs- und Wahrnehmungsstörungen. Psychometrische Korrelate im Vorschulalter. HNO 2008; 56: 638-644

19 Ptok M, Buller N. Factorial structure of the BAKO 1-4. On the validation of the "Base competence for school years 1-4" test for determining phonological processing. HNO 2006; 54 (11): 888-892

20 Ptok M, Berendes $K$, Gottal S, Grabherr B, Schneeberg J, Wittler $M$. Lese - Rechtschreibstörung. Die Bedeutung der phonologischen Informationsverarbeitung für den Schriftspracherwerb. HNO 2007; 55: $737-747$

21 Rosenauer K, Brunner M, Hornberger C, Pröschel U. Elterliche Einschätzung versus objektiver Testvefunde bei auditiver Verarbeitungs- und Wahrnehmungsstörung (AVWS) - Bestehen Zusammenhänge mit der Rechtschreibung?http://www.egms.de/en/meetings/dgpp2007/ 07dgpp83.shtml [accessed 25-3-2009]

22 Schulte-Körne G, Deimel W Bartling J, Remschmidt H. Auditory processing and dyslexia: evidence for a specific speech processing deficit. Neuroreport 1998; 9: 337-340

23 Schulte-Körne G, Deimel W, Bartling J, Remschmidt $H$. Speech perception deficit in dyslexic adults as measured by mismatch negativity (MMN). Int J Psychophysiol 2001; 40: 77-87

24 Schulte-Körne G, Remschmidt H. Legasthenie - Symptomatik, Diagnostik, Ursachen, Verlauf und Behandlung. Dtsch Ärztebl 2003; 100: 333-338

25 Task Force on Central Auditory Processing Consensus Development. Central Auditory Processing: Current Status of Research and Implications for Clinical Practice. Am J Audiol 1996; 5: 41-52

26 von Suchodoletz W. Neue Studien zeigen: Training auditiver Funktionen für sprachgestörte Kinder ohne Nutzen. Forum Logopädie 2006; 5: 18-23

27 Wohlleben B, Nubel $K$, Gross M. Orientierende Tests zur Diagnostik auditiver Verarbeitungs- und Wahrnehmungsstörungen. In: Kruse $\mathrm{E}$ Gross M, Hrsg. Aktuelle phoniatrisch-pädaudiologische Aspekte 2001/2002. 1. Auflage, Median, Heidelberg; 2001; S222-S225 